Nota de investigación

\title{
Evaluación de la deshidratación osmótica para el enriquecimiento con compuestos bioactivos en manzana
}

\author{
Francisco Xavier Osorio Gutiérrez ${ }^{1}$ \\ Alondra Peñaloza Ortiz ${ }^{1}$ \\ Yanik Ixchel Maldonado Astudillo ${ }^{1}$ \\ Javier Jiménez Hernández ${ }^{1}$ \\ Ricardo Salazar ${ }^{2 \S}$
}

${ }^{1}$ Facultad de Ciencias Químico Biológicas-Universidad Autónoma de Guerrero. Av. Lázaro Cárdenas s/n, Ciudad Universitaria Sur, Col. La Haciendita, Chilpancingo de los Bravo, Guerrero, México. CP. 39090. (checo_xavier@hotmail.com, Aloortiz348@gmail.com, yaixma@gmail.com, jjimenezuagro@gmail.com). ${ }^{2}$ CONACYT-Universidad Autónoma de Guerrero. Av. Javier Méndez Aponte núm. 1, Fracc. Servidor Agrario, Chilpancingo de los Bravo, Guerrero, México. CP. 39070.

${ }^{\S}$ Autor para correspondencia: rsalazarlo@ conacyt.mx.

\section{Resumen}

El presente estudio evalúa efecto de la deshidratación osmótica (DO) de rodajas de manzana criolla cosechada en el estado de Guerrero en una emulsión (600 g solidos $\mathrm{kg}^{-1}$ emulsión) preparada con capsul, jamaica y una oleorresina de chile piquín adurante120 min a $40{ }^{\circ} \mathrm{C}$. Además, la manzana se osmodeshidrató en una solución de sacarosa y sacarosa-jamaica para propósitos de comparación. Se analizó la influencia de la DO en la perdida de agua y en la ganancia de solidos durante el proceso. En el mismo sentido, se determinó el cambio de color, la ganancia de aceite, el contenido de fenoles solubles y antocianinas monoméricas, así como la fuerza máxima de compresión de las rodajas osmodeshidratadas liofilizadas. Las muestras tratadas con la emulsión mostraron un incremento en el contenido de aceite, fenoles solubles y antocianinas monoméricas. La presencia de jamaica en la solución osmótica favoreció el cambio de color de las muestras. Las micrografías de las rodajas osmodeshidratadas con la emulsión reveló la presencia de microcápsulas con aceite embebido en la microestructura de la manzana. Los resultaos obtenidos sugieren que esta técnica puede ser utilizada para impregnar rodajas de manzana con aceites y sustancias funcionales para producir alimentos más atractivos para el consumidor.

Palabras claves: alimentos funcionales, deshidratación osmótica, emulsión, Jamaica, manzana.

Recibido: mayo de 2019

Aceptado: junio de 2019 
La demanda y la preferencia de alimentos funcionales que proporcionen bienestar a las personas que los consumen se ha incrementado en la última década. Diversos estudios se han llevado a cabo para incorporar compuestos bioactivos para elaborar alimentos saludables mínimamente procesados. Diferentes frutas como el plátano, la fresa y el mango (Huerta-Vera et al., 2017; Jiménez-Hernández et al., 2017) han sido investigados por su idoneidad para ser enriquecidos con sustancias funcionales. En este sentido, la manzana posee un gran potencial para el fin antes mencionado por su elevada porosidad. Emser et al. (2017); Rascón et al. (2018), han enriquecido exitosamente rodajas de manzana con probióticos empleando la DO.

En la actualidad la deshidratación osmótica es considerada generalmente como un pretratamiento que puede ser usado en el procesamiento de frutas y vegetales convencionales, para mejorar su calidad y ahorrar energía (Ciurzyńska et al., 2016). La razón primordial por la que es utilizada como pretratamiento es la modificación que se logra de la composición química de las frutas y vegetales; a través, de la pérdida simultánea de agua y ganancia de sólidos solubles.

Las frutas y vegetales pueden ser transformadas en nuevos productos con relaciones diferentes entre agua y sólidos solubles, sólidos insolubles y solubles, azúcares, azúcares y ácidos, azúcares y sales (Torreggiani y Bertolo, 2001). La impregnación elevada de solutos durante la DO en la mayoría de los casos no es deseable, excepto cuando la solución osmótica posee propiedades funcionales. En este sentido, El uso de una emulsión como solución osmótica para preparar frutas osmodeshidratadas enriquecidas con compuestos bioactivos, proporciona un nuevo enfoque para el desarrollo de alimentos funcionales (Salazar, 2015).

Debido a lo anterior, el objetivo del presente trabajo fue terminar la influencia de la solución osmótica (sacarosa, sacarosa/jamaica, emulsión) en la perdida de agua (PA), ganancia de solidos (GS), contenido de aceite, color, fuerza de compresión, microestructura y la ganancia de compuestos bioactivos para explorar una metodología nueva que permita enriquecer y mejorar las rodajas de manzana a través de las sustancias funcionales de la jamaica y la oleorresina de chile piquín.

\section{Procedimientos}

Las manzanas de la variedad criolla con un estado similar de madurez, la sacarosa, el aceite de soya y el chile piquín se obtuvieron en un mercado popular de la ciudad de Chilpancingo, Guerrero.

El almidón Hi-Cap se obtuvo de Ingredion México. Todos los reactivos utilizados se adquirieron en Sigma-Aldrich (St. Louis, MO, EU) o JT Baker (Phillipsburg, NJ, EU) y fueron grado analítico. Los frutos se lavaron completamente con agua y jabón y se pelaron manualmente utilizando un pelador de acero inoxidable. Se cortaron dos rodajas paralelas a la semilla de cada fruto y luego se cortarán en rodajas de $5 \mathrm{~mm}$ empleando una cortadora afilada.

La oleorresina se prepara mezclando chile piquín molido con aceite de soya en una proporción 1:3 (p:p). en un frasco de vidrio ámbar, el cual se mantuvo durante $48 \mathrm{~h}$ para obtener el extracto oleoso. La oleorresina se decantó por gravedad, se filtró usando un tamiz (tamiz de $0.5 \mathrm{~mm}$ ) y se almaceno bajo una atmósfera de nitrógeno y se protegió de la luz a $4{ }^{\circ} \mathrm{C}$ para su uso posterior. El extracto de jamaica se preparó a partir de la decocción de $100 \mathrm{~g}$ de cálices con 1 litro de agua a $90{ }^{\circ} \mathrm{C}$ durante $10 \mathrm{~min}$. El extracto de jamaica se filtró y se almaceno a $4{ }^{\circ} \mathrm{C}$ para su uso posterior. 
Se prepararon tres soluciones osmóticas. La solución de sacarosa se obtuvo disolviendo $600 \mathrm{~g}$ de azúcar refinado en $400 \mathrm{~mL}$ de agua destilada. La solución de sacarosa-jamaica se preparó disolviendo $600 \mathrm{~g}$ de azúcar refinado en $400 \mathrm{~mL}$ del extracto de jamaica mencionado anteriormente. La emulsión se obtuvo mezclando $60 \mathrm{~g}$ de almidón Hi-Cap, $20 \mathrm{~g}$ de olerorresina de chile piquín, 530 de sacarosa y 400 g de agua.

Las rodajas de manzana se osmodeshidrataron en las soluciones osmóticas utilizando una relación 1:30 (p:p) fruta: solución durante 120 min a $40{ }^{\circ} \mathrm{C}$. Las muestras se extrajeron a $15,20,45,60,75$, 90, 105 y 120 min y el exceso de solución de la superficie se retiró utilizando toallas de papel. La pérdida de agua y la ganancia de solidos se calculó utilizando el método continuo propuesto por Azuara (1998).

Posteriormente las muestras osmodeshidratadas se liofilizaron durante 4 días y se determinó el contenido de aceite (método AOAC 934.01), la humedad (método AOAC 960.39), el color (Jiménez-Hernández et al., 2017), el contenido de fenoles solubles (De La Parra, 2007) y el contenido de antocianinas monoméricas (Sáyago-Ayerdi, 2013). La microestructura de las muestras osmodeshidratadas y liofilizadas se observó en un microscopio electrónico de barrido (ESEM; Philips modelo XL30). La fuerza máxima de compresión se midió en un analizador de textura TA-XT2. Todos los resultados se expresaron como promedios $(n=3)$. Un análisis de varianza de una sola vía a través de un diseño completamente al azar. Las diferencias entre los diferentes grupos se determinaron con la prueba de Tukey. Todos los análisis estadísticos se llevaron a cabo con el paquete JMP 9.0 (SAS Institute Inc., Cary, NC) en el nivel de significancia de $\alpha=0.05$.

\section{Discusión}

La Figura 1 y 2 muestran el efecto de las soluciones osmóticas empleadas durante la DO de las rodajas de manzana en la perdida de agua (PA) y en la ganancia de solidos (GS). La pérdida de agua después de 120 min para las rodajas de manzana tratadas en la solución de sacarosa, sacarosajamaica y emulsión fue de $4.42 \pm 0,0.37 \pm 0.01$ y $0.36 \pm 0.01 \mathrm{~g}$ agua $\mathrm{g}^{-1}$ fruta fresca, respectivamente. Por otro lado, los valores de la ganancia de solidos después de 120 min para las rodajas de manzana tratadas en la solución de sacarosa, sacarosa-jamaica y emulsión fueron de $0.08 \pm 0,0.08 \pm 0.01$ y $0.04 \pm 0.01 \mathrm{~g}$ sólidos $\mathrm{g}^{-1}$ fruta fresca. Cada tipo de solución osmótica tuvo un potencial similar $(p<$ 0.05) para remover agua. En contraste, durante la DO con la emulsión, la cantidad de solidos ganados mostró un comportamiento diferente a la solución de sacarosa y sacarosa-jamaica. Cuando se utilizó la solución de sacarosa y sacarosa-jamaica, se obtuvieron valores similares de PA y G. Esta diferencia puede explicarse con base en el tamaño de las moléculas de almidón empleadas en la emulsión, las cuales bloquean los poros de la superficie de la manzana, disminuyendo los sólidos impregnados para favorecer la PA por difusión.

Las propiedades físicas y químicas de las rodajas de manzana al final del tratamiento osmótico se muestran en el Cuadro 1. Como puede observarse el uso de la jamaica como agente osmótico tuvo un efecto favorable en el contenido de fenoles solubles y antocianinas monoméricas de las rodajas de manzana procesadas. Se obtuvo un incremento de 79 y $145 \%$ de fenoles solubles en las muestras deshidratadas en la solución sacarosa-jamaica y emulsión, respectivamente. En el mismo sentido, se obtuvo un incremento significativo $(p<0.05)$ en el contenido de antocianinas monoméricas. 


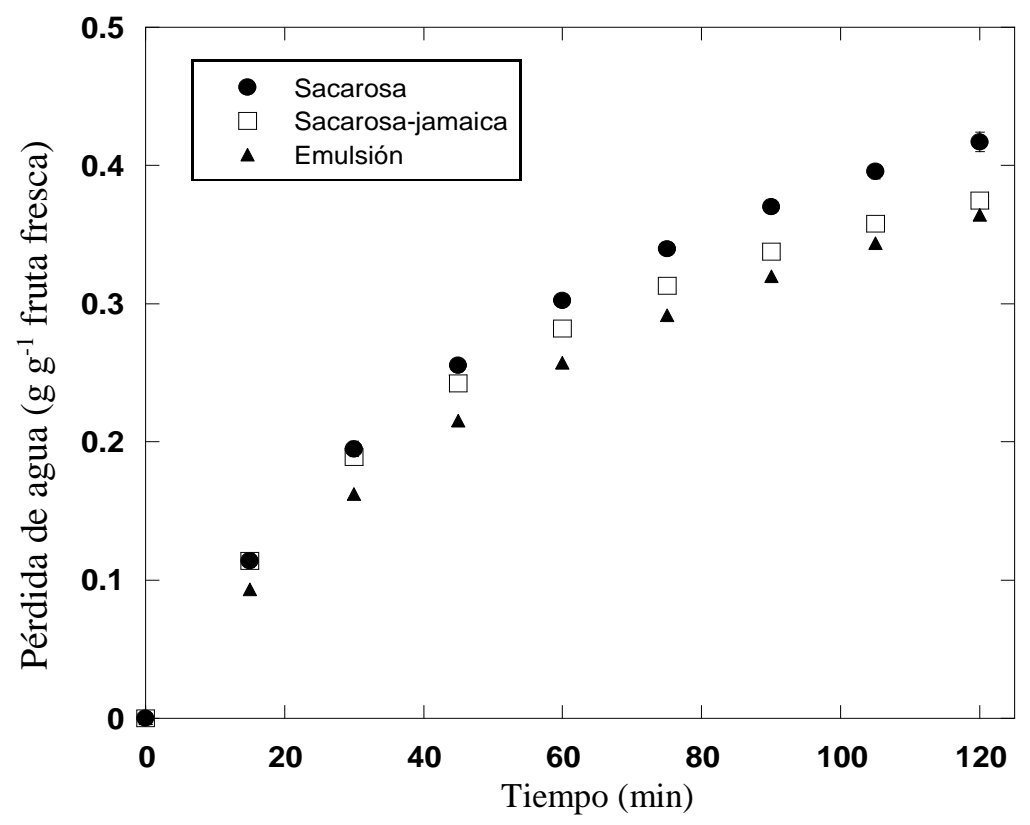

Figura 1. Pérdida de agua durante la deshidratación osmótica de rodajas de manzana a $40{ }^{\circ} \mathrm{C}$.

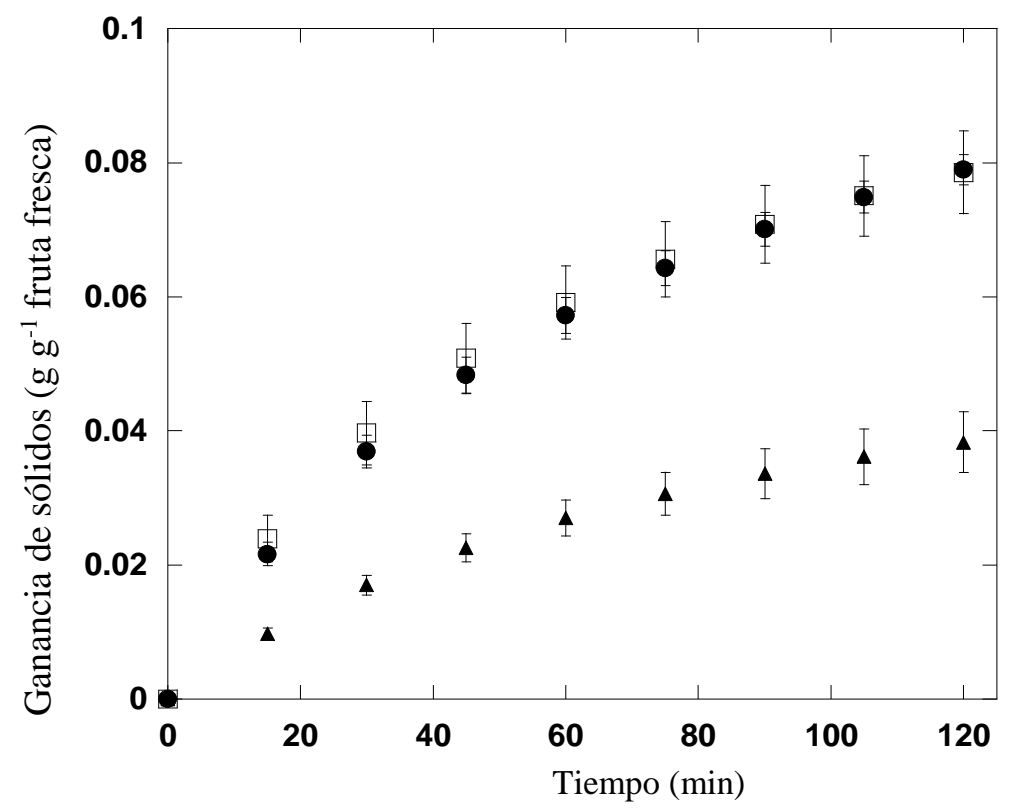

Figura 2. Ganancia de sólidos durante la deshidratación osmótica de rodajas de manzana a $40{ }^{\circ} \mathrm{C}$.

Los cálices de jamaica son una fuente importante de antioxidantes naturales como las antocianinas y diversos compuestos fenólicos debido a su elevado contenido de pigmentos de color rojo (CidOrtega y Guerrero-Beltrán, 2015). Los cambios de color son unos de los cambios más significativos observados durante la DO. Como puede ser observado en el Cuadro 1, las rodajas de manzana mostraron un cambio significativo en su coloración. 
Cuadro 1. Propiedades físicas y químicas de las rodajas de manzana osmodeshidratadas durante 120 min a $40{ }^{\circ} \mathrm{C}$ y liofilizadas.

\begin{tabular}{lccccc}
\hline Solución osmótica & $\begin{array}{c}\text { Fenoles } \\
\text { solubles } \\
\left(\mathrm{mg} \mathrm{g}^{-1}\right)\end{array}$ & $\begin{array}{c}\text { Antocianinas } \\
\text { monoméricas } \\
\left(\mathrm{mg} \mathrm{g}^{-1}\right)\end{array}$ & $\begin{array}{c}\text { Ángulo matiz } \\
\left(^{\circ}\right)\end{array}$ & $\begin{array}{c}\text { Fuerza máxima } \\
\text { de compresión } \\
(\mathrm{N})\end{array}$ & $\begin{array}{c}\text { Aceite } \\
\text { impregnado } \\
(\%)\end{array}$ \\
\hline Sacarosa & $5.64 \pm 0.73 \mathrm{~b}$ & $\mathrm{nd}$ & $82.1 \pm 3.2 \mathrm{a}$ & $247.6 \pm 29.06 \mathrm{a}$ & $0.1 \pm 0 \mathrm{c}$ \\
Sacarosa-jamaica & $10.13 \pm 0.3 \mathrm{a}$ & $0.07 \pm 0.01 \mathrm{a}$ & $19.96 \pm 7.01 \mathrm{~b}$ & $271.5 \pm 27.65 \mathrm{a}$ & $0.6 \pm 0.3 \mathrm{~b}$ \\
Emulsión & $13.86 \pm 0.6 \mathrm{a}$ & $0.11 \pm 0.03 \mathrm{a}$ & $23.5 \pm 5.73 \mathrm{~b}$ & $268.38 \pm 37.02 \mathrm{a}$ & $4.33 \pm 0.01 \mathrm{a}$ \\
\hline
\end{tabular}

Promedio \pm desviación estándar. Los promedios seguidos de una letra diferente, en la misma columna, son significativamente diferentes; $n d=$ no detectado.

La presencia de la jamaica y la oleorreina favorecieron el cambio de color de las rodajas de manzana de un a un tono rojizo con respecto a las rodajas procesadas en la solución de sacarosa (tono amarillo). Es importante resaltar, que el empleo de la emulsión como agente osmótico incremento significativamente el contenido de aceite en las rodajas de manzana. Al final de la DO, la emulsión produjo rodajas de manzana con un contenido de aceite de $4.33 \mathrm{~g}$ aceite $100 \mathrm{~g}^{-1} \mathrm{de}$ fruta seca. Resultados similares han sido descritos para piña y mango, cuando se utilizaron emulsiones preparadas con goma arábiga, inulina y oleorresina de chile piquin (Salazar-López et al., 2015; Jiménez-Hernández et al., 2017).

Los resultados en este estudio sugieren que el uso de un extracto acuoso de jamaica y la oleorresina de chile piquín como agentes osmóticos, es una manera sencilla para incrementar el contenido de compuestos bioactivos y modificar el color de frutas y verduras durante la DO para crear alimentos más atractivos y saludables para el consumidor. No se observaron cambios significativos $(p<0.05)$ en la fuerza máxima de compresión de las rodajas de manzana osmodeshidratadas y liofiizadas.

Lo anterior indica, que ninguno de los ingredientes utilizados tuvo un impacto en la textura de las rodajas de manzana procesadas. Por último, los cambios estructurales de las rodajas de manzana después de la DO y el liofilizado se observaron en un microscopio electrónico de barrido (Figura 3). Durante la DO las células redujeron su forma y su tamaño por la pérdida de agua provocando un encogimiento del tejido celular. Independientemente, de la solución osmótica utilizada, se observó una superficie lisa. Sin embargo, la DO con emulsiones hizo posible incorporar microcápsulas de almidón y la oleorresina de chile piquín sobre el tejido de las rodajas de manzana.

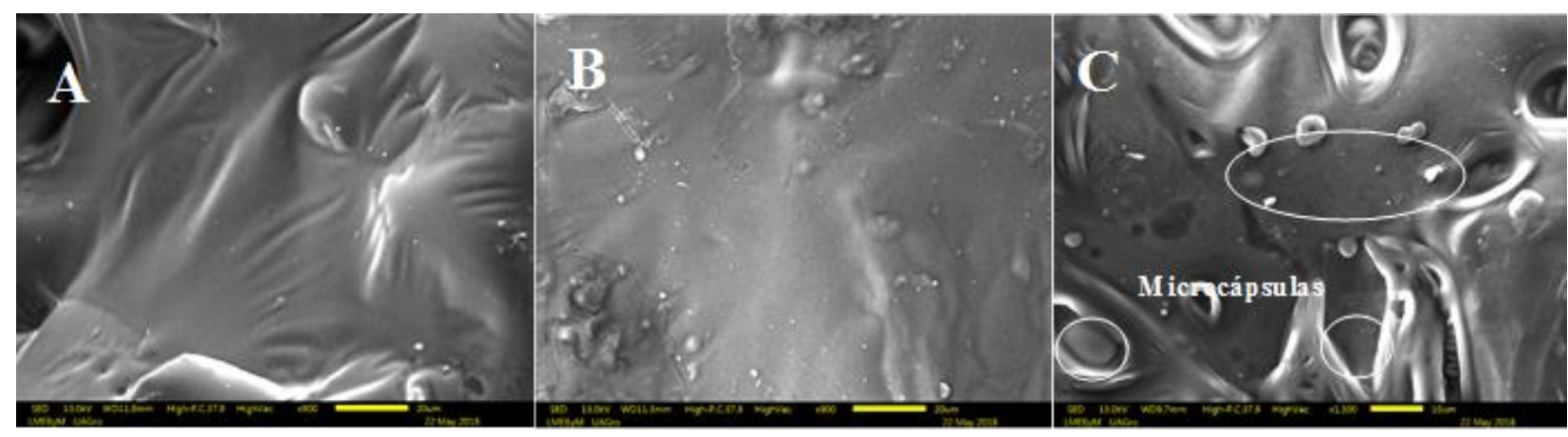

Figura 3. Micrografías de las rodajas de manzana osmodeshidratadas durante 120 min a $40{ }^{\circ} \mathrm{C}$ y liofilizadas. (A) sacarosa; (b) sacarosa-jamaica; y (c) emulsión. 


\section{Conclusiones}

El presente estudio exploró el potencial de la DO con emulsiones para incorporar compuestos bioactivos en rodajas de manzana. Los resultados obtenidos mostraron que el uso de un extracto acuoso de jamaica como agente osmótico tiene el potencial para modificar el color e incrementar el contenido de fenoles solubles y las antocianinas monoméricas. En el mismo sentido, la emulsión preparada con jamaica y la oleorresina de chile piquín permitió obtener rodajas de manzana con un contenido significativamente mayor de aceite por la incorporación de microcápsulas de almidón y la oleorresina de chile piquín sobre el tejido de las rodajas de manzana. La metodología mostrada en este trabajo puede ser utilizada para mejorar el sabor y el color de la manzana a través de la impregnación con aceites y compuestos con atributos funcionales.

\section{Literatura citada}

Azuara, E.; Beristain, C. I. and Gutiérrez, G. F. 1998. A method for continuous kinetic evaluation of osmotic dehydration. LWT. Food Sci. Technol. 31(4):317-321.

Cid-Ortega, S. and Guerrero-Beltrán, J. A. 2015. Roselle calyces (Hibiscus sabdariffa), an alternative to the food and beverages industries. J. Food Sci. Technol. 52(11):6859-6869.

Ciurzyńska, A.; Kowalska, H.; Czajkowska, K. and Lenart, A. 2016. Osmotic dehydration in production of sustainable and healthy food. Trends Food Sci. Technol. 50(1):186-192.

De La Parra, C.; Serna Saldivar, S. O. and Liu, R. H. 2007. Effect of processing on the phytochemical profiles and antioxidant activity of corn for production of masa, tortillas, and tortilla chips. J. Agric. Food Chem. 55(10):4177-4183.

Emser, K.; Barbosa, J.; Teixeira, P. and Bernardo de Morais, A. M. M. 2017. Lactobacillus plantarum survival during the osmotic dehydration and storage of probiotic cut apple. Journal of Functional Foods. 38(1):519-528.

Huerta-Vera, K.; Flores-Andrade, E.; Pérez-Sato, J. A.; Morales-Ramos, V.; Pascual-Pineda, L. A.; and Contreras-Oliva, A. 2017. Enrichment of Banana with Lactobacillus rhamnosus using double emulsion and osmotic dehydration. Food Bio. Technol. 10(6):1053-1062.

Jiménez-Hernández, J.; Estrada-Bahena, E. B.; Maldonado-Astudillo, Y. I.; Talavera-Mendoza, Ó.; Arámbula-Villa, G.; Azuara, E. and Salazar, R. 2017. Osmotic dehydration of mango with impregnation of inulin and piquin-pepper oleoresin. LWT. Food Sci. Technol. 79(1):609-515.

Rascón, M. P.; Huerta-Vera, K.; Pascual-Pineda, L. A.; Contreras-Oliva, A.; Flores-Andrade, E.; Castillo-Morales, M. and González-Morales, I. 2018. Osmotic dehydration assisted impregnation of Lactobacillus rhamnosus in banana and effect of water activity on the storage stability of probiotic in the freeze-dried product. Lwt. 92(3):490-496.

Salazar-López, E. I.; Jiménez, M.; Salazar, R. and Azuara, E. 2015. Incorporation of microcapsules in pineapple intercellular tissue using osmotic dehydration and microencapsulation method. Food Bio. Technol. 8(8):1699-1706.

Sáyago-Ayerdi, S. G.; Velázquez-López, C.; Montalvo-González, E. and Goñi, I. 2013. By-product from decoction process of Hibiscus sabdariffa L. calyces as a source of polyphenols and dietary fiber. J. Sci. Food Agric. 94(5):898-904.

Torreggiani, D. and Bertolo, G. 2001. Osmotic pre-treatments in fruit processing: chemical, physical and structural effects. J. Food Eng. 49(2-3):247-253. 